\title{
A cumulative effect involving malfunction of the PTH1R and $A T P 4 A$ genes explains a familial gastric neuroendocrine tumor with hypothyroidism and arthritis
}

\author{
Oriol Calvete ${ }^{1,2} \cdot$ Maite Herraiz $^{3} \cdot$ Jose Reyes $^{4} \cdot$ Ana Patiño $^{5} \cdot$ Javier Benitez $^{1,2}$
}

Received: 9 February 2017/ Accepted: 23 April 2017/Published online: 4 May 2017

(c) The International Gastric Cancer Association and The Japanese Gastric Cancer Association 2017

\begin{abstract}
Background Type I gastric neuroendocrine tumors (gNETs) classically arise because of hypergastrinemia and involve destruction of parietal cells, which are responsible for gastric acid secretion through the ATP4A proton pump and for intrinsic factor production.

Methods By whole exome sequencing, we studied a family with three members with gNETs plus hypothyroidism and rheumatoid arthritis to uncover their genetic origin.

Results A heterozygous missense mutation in the ATP4A gene was identified. Carriers of this variant had low ferritin and vitamin $\mathrm{B}_{12}$ levels but did not develop gNETs. A second heterozygous mutation was also uncovered (PTHIR p.E546K). Carriers exhibited hypothyroidism and one of them had rheumatoid arthritis. Gastrin activates parathyroid hormone like hormone/parathyroid hormone 1 receptor (PTH1R) signaling, which is involved in gastric cell
\end{abstract}

Electronic supplementary material The online version of this article (doi:10.1007/s10120-017-0723-8) contains supplementary material, which is available to authorized users.

Javier Benitez

jbenitez@cnio.es

1 Human Genetics Group, Spanish National Cancer Research Center (CNIO), Melchor Fernández Almagro 3, 28029 Madrid, Spain

2 Biomedical Research Networking Center on Rare Diseases (CIBERER), 28029 Madrid, Spain

3 Department of Gastroenterology, University Clinic of Navarra, 31008 Pamplona, Spain

4 Department of Gastroenterology, Hospital INCA, 07300 Majorca, Spain

5 Department of Pediatrics and Clinical Genetics Unit, University Clinic of Navarra, 31008 Pamplona, Spain homeostasis. Activation of parathyroid hormone/PTH1R, which is upregulated by thyrotropin in the thyroid, is also involved in $R A N K L$ expression, which regulates bone homeostasis. Thyrotropin and $R A N K L$ expression were deregulated in $P T H I R$ mutation carriers, suggesting a link between the PTHIR gene, hypothyroidism, rheumatoid arthritis, and gastric disease. Only patients with both mutations developed gNETs plus hypothyroidism and rheumatoid arthritis.

Conclusion Both mutations suggest that a collaborative mechanism is operative in this family, in which mutations in these genes affect the function and viability of parietal cells and lead to the achlorhydria that drives hypergastrinemia and the formation of gNETs.

Keywords Gastric neuroendocrine tumor - PTHIR . ATP4A $\cdot$ Hypothyroidism $\cdot$ Rheumatoid arthritis

\section{Introduction}

Type I gastric neuroendocrine tumors (gNETs) arise from gastric enterochromaffin-like cells. They have a benign course and a late age of onset (mean 66 years). Classically, hypergastrinemia in patients who have autoimmune atrophic gastritis causes hyperplasia of gastric enterochromaffin-like cells that can progress to type I gNETs [1]. Tumor progression is associated with the destruction of parietal cells, which are responsible for gastric acid and intrinsic factor secretion, and leads to hypochlorhydria and vitamin $\mathrm{B}_{12}$ malabsorption anemia caused by a lack of gastric acid and intrinsic factor respectively [2]. In normal conditions, gastrin directly regulates acid secretion of parietal cells, the proliferation, development, and 
homeostasis of which are coordinated by the acidity of the stomach and different growth factors (Fig. 1a).

Parathyroid hormone like hormone (PTHLH) has been identified as one of these gastric growth factors, and responds to gastrin/cholecystokinin B receptor 2 (CCKR2) activation [3]. PTHLH interacts with the parathyroid hormone 1 receptor (PTH1R) to activate different growth factors involved in gastric homeostasis and proliferation (Fig. 1b). In Cckbr (gastrin receptor) knockout mice, PTHLH is downregulated [4].

PTH1R is also found in the thyroid gland, and responds to parathyroid hormone (PTH) activation, which is regulated by thyrotropin (Fig. 1b). PTH/PTH1R activation is involved in osteoclast production and bone development [5]. Both osteopenia/osteoporosis and thyroid malfunction were related with chronic atrophic gastritis, hypergastrinemia, and hypoacidity [6, 7]. In particular, malabsorption of vitamin $\mathrm{B}_{12}$ (anemia due to a lack of intrinsic factor) has been reported in several hypothyroidism disorders. However, although various bone and thyroid diseases are clinically related to gastric disease, a common genetic origin has not been elucidated.

Recently, we studied a consanguineous family (family 1)with five members affected by type I gNETs (Table 1) [8]. We identified the deleterious p.R703C mutation in homozygosis in the ATP4A gene, which explained the lack of acid secretion and ferropenic anemia and the origin of hypergastrinemia and gNET development instead of a classic tumorigenesis process $[8,9]$.

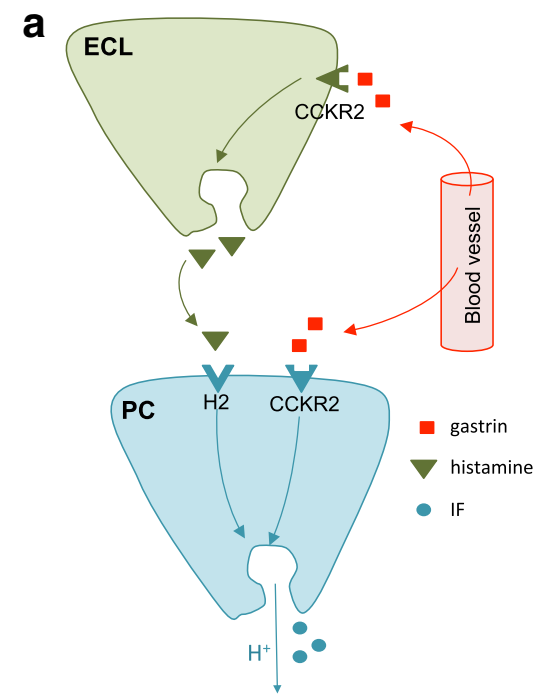

Fig. 1 Regulation of acid and intrinsic factor secretion and gastric homeostasis. a Gastrin acts directly on enterochromaffin-like (ECL) cells and parietal cells through cholecystokinin B receptor 2 (CCKR2). Histamine from ECL cells also positively regulates the activity of parietal cells through $\mathrm{H}_{2}$ receptors. b Left Activation of gastrin/CCKR2 regulates parathyroid hormone like hormone
In the present study we performed Whole exome sequencing of a second family from Navarra (Spain) (family 2) with 11 siblings, including three members with gNETs (45 years old on average at diagnosis); these patients had classical clinical features, including low levels of ferritin and vitamin $B_{12}$-deficiency anemia (Fig. 2a), but also had hypothyroidism and high thyrotropin levels (Table 1). Rheumatoid arthritis was also diagnosed in affected member II.10 at an age of 38 years. The mother also had hypothyroidism and rheumatoid arthritis.

\section{Materials and methods}

\section{Whole exome sequencing and bioinformatics pipeline}

Genomic DNA was isolated from peripheral blood lymphocytes with a FlexiGene DNA kit (QIAGEN). Exomes from selected DNA samples were fully captured, enriched, and sequenced (see the electronic supplementary material).

\section{Immunohistochemistry}

Paraffin-embedded tissue samples were obtained from total gastrectomy specimens and monitoring biopsies (Fig. 2a). Anti-intrinsic factor antibody was produced at the Spanish National Cancer Research Center (Table S1). b

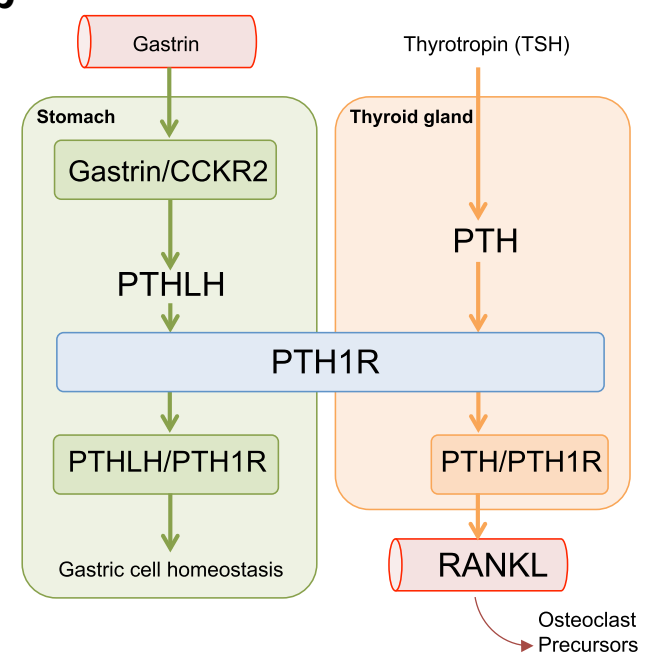

(PTHLH)/parathyroid hormone 1 receptor (PTH1R) activation, which is involved in gastric cell homeostasis and proliferation. Right Thyrotropin (TSH) promotes parathyroid hormone (PTH) secretion in the thyroid gland. Activation of PTHLH/PTH1R is involved in bone homeostasis through receptor activator of nuclear factor $\kappa \mathrm{B}$ ligand (RANKL) regulation. $I F$ intrinsic factor, $P C$ parietal cell 
Table 1 Mean age at onset and average serum concentrations of gastrin, vitamin $\mathrm{B}_{12}$, ferritin, and thyrotropin of members of families 1 and 2 with gastric neuroendocrine tumors (gNETs) and ATP4A p.Q680L carriers

\begin{tabular}{lllllll}
\hline Family & $\begin{array}{l}\text { gNET members/total } \\
\text { members }\end{array}$ & $\begin{array}{l}\text { Mean age at diagnosis } \\
\text { (years) }\end{array}$ & $\begin{array}{l}\text { Gastrin } \\
(0-100 \mathrm{pM})\end{array}$ & $\begin{array}{l}\text { Vitamin } \mathrm{B}_{12} \\
(210-920 \mathrm{pg} / \mathrm{mL})\end{array}$ & $\begin{array}{l}\text { Ferritin } \\
(25-28 \mathrm{ng} / \mathrm{mL})\end{array}$ & $\begin{array}{l}\text { Thyrotropin } \\
(0.3-5.5 \mathrm{IU} / \mathrm{mL})\end{array}$ \\
\hline Family 1 & $5 / 10$ & 35 & $484 \mathrm{pM}$ & $444.25 \mathrm{pg} / \mathrm{mL}$ & $6.25 \mathrm{ng} / \mathrm{mL}$ & $0.75 \mathrm{IU} / \mathrm{mL}$ \\
Family 2 & $3 / 11$ & 45 & $1819 \mathrm{pM}$ & $105.00 \mathrm{pg} / \mathrm{mL}$ & $13.50 \mathrm{ng} / \mathrm{mL}$ & $9.62 \mathrm{IU} / \mathrm{mL}$ \\
\hline
\end{tabular}

Normal levels are shown in parentheses

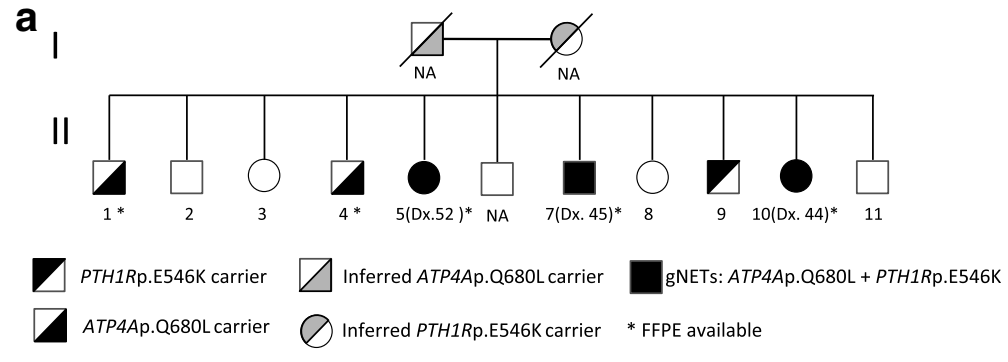

Dx. Age of onset (gNET cases) NA: DNA samples not available

\section{C}

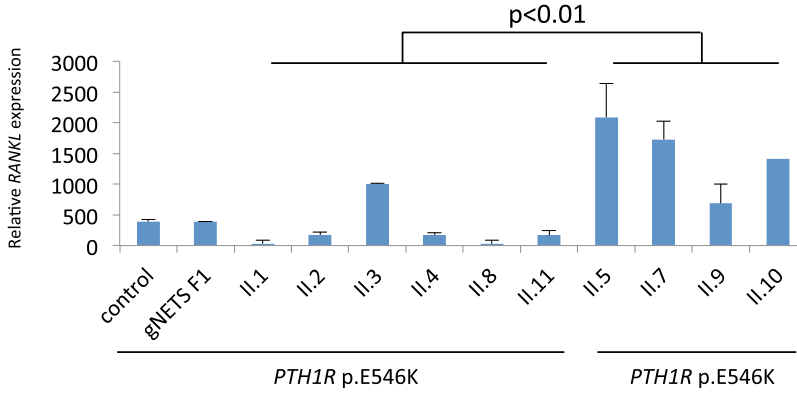

Fig. 2 a Pedigree of family 2. Age of onset [gastric neuroendocrine tumor $(g N E T)$ cases] is shown in parentheses. Segregation for the ATP4A p.Q680L and PTH1R p.E546K mutations found in this work is also shown. The genotype of the parents is inferred. An asterisk indicates that formalin-fixed and paraffin-embedded (FFPE) tissue was available. b Hybridization with anti-intrinsic factor antibody to normal stomach wild-type $(w t)$. Lower staining with anti-intrinsic factor was observed in II.4, who carries only the ATP4A mutation.

\section{Real-time quantitative PCR}

Real-time quantitative PCR was performed with complementary DNA to test the levels of expression of PTHIR messenger RNA. Complementary DNA was obtained by reverse transcription PCR of $1200 \mathrm{ng}$ of total RNA from peripheral blood cells with use of a high-capacity complementary DNA reverse transcription kit (Applied Biosystems catalog no. 4368814) following the manufacturer's instructions. PCR was performed with complementary DNA at approximately $25 \mathrm{ng} / \mathrm{mL}$ and Power SYBR Green PCR master mix (Applied Biosystems cata$\log$ no. 4367659). Expression levels were evaluated with

\section{Results}

b
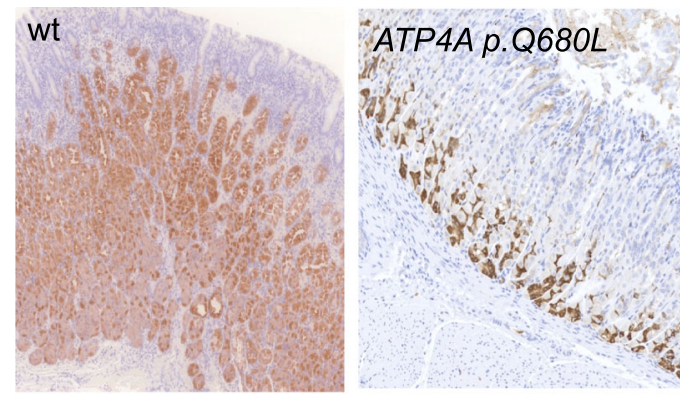

gNETs. ATP4A p.Q680L + PTH1R p.E546K

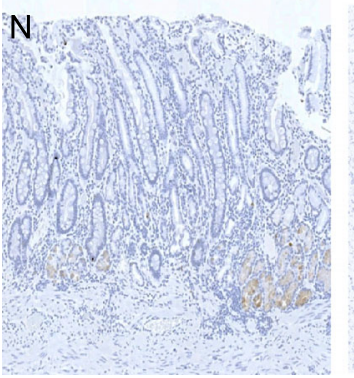

One gNET individual (II.7) is also shown as an example. Normal tissue $(N)$ and tumor tissue $(T)$ from gNET individuals were evaluated separately. c Reverse transcription PCR studies of RANKL expression in serum. The members studied are grouped according to PTH1R p.E546K mutation. Student's $t$ test was used for the statistical analysis of normally distributed values. RANKL expression with normalized using GAPDH messenger RNA levels as a control. $F 1$ family 1

the $\Delta \Delta C_{\mathrm{t}}$ method [10] and normalized with use of $G A P D H$ quantification as a standard.

We first studied the whole ATP4A gene by Sanger sequencing (Table S2). A heterozygous deleterious mutation (ATP4A p.Q680L; rs61729956) was found in the three affected members with gNETs (Fig. 2a, Table 2). The p.Q680 position is located in the same phosphoryl-binding pocket as the ATP4A p.R703C mutation that caused the gNETs in family 1 [8]. This mutation may affect the 
Table 2 Variants found in the ATP4A and PTH1R genes for families F1 and F2

\begin{tabular}{|c|c|c|c|c|c|c|c|c|c|}
\hline \multirow[t]{2}{*}{ Family } & \multirow[t]{2}{*}{ Gene } & \multirow[t]{2}{*}{ Exon } & \multirow{2}{*}{$\begin{array}{l}\text { Complementary } \\
\text { DNA }\end{array}$} & \multirow[t]{2}{*}{ Protein } & \multirow{2}{*}{$\begin{array}{l}\text { Damage } \\
\text { prediction }\end{array}$} & \multirow[t]{2}{*}{ Inheritance } & \multirow[t]{2}{*}{ dbSNP } & \multicolumn{2}{|l|}{ Frequency } \\
\hline & & & & & & & & $\begin{array}{l}1000 \\
\text { Genomes }\end{array}$ & ExAc \\
\hline Family 1 & ATP4A & 14 & c. $2107 \mathrm{C}>\mathrm{T}$ & p.R703C & Deleterious & Homozygous & Calvete et al. [8] & ND & ND \\
\hline \multirow[t]{2}{*}{ Family 2} & ATP4A & 14 & c. $2039 \mathrm{~A}>\mathrm{T}$ & p.Q680L & Deleterious & Heterozygous & rs61729956 & 0.003 & 0.003 \\
\hline & PTH1R & 14 & c. $1636 \mathrm{G}>\mathrm{A}$ & p.E546K & Deleterious & Heterozygous & rs77048718 & 0.008 & 0.016 \\
\hline
\end{tabular}

$N D$ not described

ATP4A protein in a similar way as the mutation in family 1 and affect the effectiveness of gastric acid production [9]. Two other siblings who did not have gNETs (II.1 and II.4) also carried this variant (Fig. 2a). Carriers of this mutation have an intermediate phenotype; these individuals had low ferritin $(20.7 \mathrm{ng} / \mathrm{mL})$ and vitamin $\mathrm{B}_{12}(187.9 \mathrm{pg} / \mathrm{mL})$ levels, although these levels were not as low as in the gNET patients (Table 1) and were not sufficiently low for them develop gNETs.

Whole exome sequencing was performed in two affected siblings with type I gNETs (II.7 and II.10), looking for a second gene. A deleterious missense mutation (rs77048718) in the PTH1R gene (p.E546K) was uncovered (Table 2). PTH1R encodes the transmembrane receptor for PTH and PTHLH, which is related to the development of parietal cells and the stomach (Fig. 1b). By in silico studies we compared the putative PTH1R p.E546K protein with the wild-type PTH1R (Fig. S1). Putative binding sites were annotated with the standard score given by PredictProtein (threshold greater than 20) (Table S3) [11]. Five of 15 putative binding sites were reduced (threshold less than 20) in the putative abnormal protein, including an RNA binding site (Fig. S1). In addition, three affected members had hypothyroidism and at least one of them also had rheumatoid arthritis (II.10). Although we were not able to study the genetic condition of the parents, the mother also had hypothyroidism and rheumatoid arthritis.

Sanger sequencing confirmed that the three members with gNETs shared two mutations (ATP4A p.Q680L and PTH1R p.E546K) (Fig. 2a). Both mutations, which belong to the same regulation pathway for parietal homeostasis (Fig. 2b), have an expected frequency of being together of $0.0024 \%$.

\section{Immunohistochemistry studies}

Anti-intrinsic factor, which stains parietal cells, was hybridized to the stomach of ATP4A p.Q680L mutation carriers and the gNET patients. Lower immunoreactivity was observed for individuals carrying this mutation compared with normal (wild-type) stomachs (Fig. 2b). The less intense staining of parietal cells is in agreement with low ferritin and vitamin $B_{12}$ levels, and demonstrates the deleterious effect of this mutation on gastric acid secretion and homeostasis of the stomach. Positive staining was not observed with anti-intrinsic factor in the tumor tissue of the affected members from family 2 (Fig. 2b). Lower staining of gNETs compared with tissue from carriers of the ATP4A p.Q680L mutation alone suggests a cumulative effect with the PTH1R p.E546K mutation.

\section{RANKL expression}

PTH1R is also involved in the regulation of calcium and bone formation. Thyrotropin regulates activation of PTH/ PTH1R to maintain the osteoclast/osteoblast equilibrium through receptor activator of nuclear factor $\kappa \mathrm{B}$ ligand (RANKL) (Fig. 1b). Therefore, high thyrotropin levels found in gNET patients (Table 1) might be explained by the lack of negative regulation through PTH/PTH1R activation of this stimulating hormone (Fig. 1b). To evaluate the putative effect of the PTH1R p.E546K mutation, RANKL expression levels were also studied by real-time quantitative PCR in peripheral blood, as PTH1R regulates RANKL expression. Relative RANKL messenger RNA levels were increased in the three gNET individuals of family 2 (Fig. 2c). RANKL expression was also increased in the member carrying the PTH1R mutation alone and who did not have gNETs (II.9). No different expression of $R A N K L$ was observed in these gNET members from family $\mathrm{F} 1$; this suggests that the altered expression depends on the mutation of PTHIR and not on the achlorhydria or any other clinical factor of gNETs. Importantly, thyrotropin levels were never found increased in gNET members of family 1 (Table 1 ).

\section{Discussion}

Heterozygous carriers of the ATP4A p.Q680L mutation (II.1 and II.4) exhibited slightly decreased gastric acid secretion. On the other hand, PTH1R is activated by 
PTHLH in the stomach and directly regulates gastric mucosa development (Fig. 1b) [3]. PTHLH expression depends on transcription factors activated by gastrinCCKR2, since it is downregulated in $C c k b r$-knockout mice, which lack gastric acid secretion [4]; this demonstrates that PTHLH/PTH1R activation is involved in gastric chlorhydria homeostasis. Therefore, carriers of only one mutation had fewer functional parietal cells and an intermediate phenotype. Importantly, none of the members who carried only one of the ATP4A p.Q680L or PTHIR p.E546K mutations developed gNETs. Both heterozygous mutations partially contribute to parietal cell malfunction in a collaborative manner, which would accumulate the malfunction of the two genes (Fig. 1b). Members of this family with gNETs had both pathogenic mutations in the ATP4A and $P T H 1 R$ genes. Although the mutations were annotated in two different genes, both are related to the viability and function of parietal cells and gastric homeostasis (Fig. 2a) that correlate with a lack of chlorhydria and intrinsic factor secretion, which would explain iron-deficiency anemia and vitamin B12-deficiency anemia.

PTH also activates PTH1R in the thyroid gland (Fig. 1b). Carriers of the PTHIR p.E546K mutation from family 2 had increased levels of thyrotropin (Table 1), which regulates parathyroid cell activity and RANKL expression (Fig. 2c), and which also depends on PTH/ PTH1R activation. The expression of RANKL was also found to be altered when a short hairpin RNA was used to reduce PTH1R expression in cells from a murine osteosarcoma model [12], suggesting that in our case the altered expression of RANKL was correlated with the PTHIR mutation. High levels of thyrotropin and RANKL have been described in nongastric diseases such as Hashimoto thyroiditis [13] and rheumatoid arthritis [14] respectively. These altered thyrotropin and RANKL levels can explain the hypothyroidism and rheumatoid arthritis respectively observed in the family. The frequency of the PTH1R mutation is 0.008 (Table 2), and we cannot discard incomplete penetrance regarding rheumatoid arthritis and thyroid malfunction. The risk of developing rheumatoid arthritis throughout life in adults is $3.6 \%$ for women and $1.7 \%$ for men [15]. In addition, rheumatoid arthritis has a late age of onset (65 years on average), which also might hide its real prevalence.

Whole clinical data and DNA from the parents were not available. Both diseases (Hashimoto thyroiditis and rheumatoid arthritis) were observed in the mother. Therefore, we can speculate that PTHIR p.E546K mutation segregated from the mother. As far as we know, none of the parents developed gNETs. Only combination with the mutation ATP4A p.Q680L mutation started the tumorigenesis process in this family. Therefore, the ATP4A p.Q680L allele would segregate from the father.
All these data suggest a cumulative mechanism involving two genes in gNETs in this family and for the first time involving the PTHIR gene. Therefore, lack of chlorhydria leads to hypergastrinemia and gNETs that differ from the classic description of tumorigenesis of gNETs, where hypergastrinemia leads to the destruction of parietal cells that results in hypochlorhydria [2]. Mutation in the PTHIR gene also explains hypothyroidism and arthritis. Various bone and thyroid diseases have been clinically related to gastric disease [6,7], but this is the first time that these different diseases have been related to a common genetic background.

\section{Compliance with ethical standards}

Conflict of interest The authors declare that they have no conflict of interest.

Ethics approval All procedures followed were in accordance with the ethical standards of the responsible committee on human experimentation (institutional and national) and with the Helsinki Declaration of 1964 and later versions.

Informed consent Informed consent was obtained from all patients included in the study.

Funding Javier Benitez's laboratory is partially funded by the Spanish Center for Biomedical Network Research on Rare Diseases (CIBERER), the H2020 BRIDGES project, and the Spanish Ministry of Health (PI12/00070 and PI16/00440).

\section{References}

1. Coati I, Fassan M, Farinati F, Graham DY, Genta RM, Rugge M. Autoimmune gastritis: pathologist's viewpoint. World J Gastroenterol. 2015;21(42):12179-89.

2. Pritchard DM, Berry D, Przemeck SMC, Campbell F, Edwards SW, Varro A. Gastrin increases mcl-1 expression in type I gastric carcinoid tumors and a gastric epithelial cell line that expresses the CCK-2 receptor. Am J Physiol Gastrointest Liver Physiol. 2008;295:G798-805.

3. Liu C, Chen J, Guo Y, Yang L, Zhao C, Bai L. The expression of PTHLH in human gastric mucosa enterochromaffin-like cells. Dig Dis Sci. 2011;56:993-8.

4. Zhao CM, Kodama Y, Flatberg A, Beisvag V, Kulseng B, Sandvik AK, et al. Gene expression profiling of gastric mucosa in mice lacking CCK and gastrin receptors. Regul Pept. 2014;192:35-44.

5. Wysolmerski JJ. Parathyroid hormone-related protein: an update. J Clin Endocrinol Metab. 2012;97:2947-56.

6. Aasarød KM, Mosti MP, Stunes AK, Reseland JE, Basso T, Syversen U, et al. Impaired skeletal health in patients with chronic atrophic gastritis. Scand J Gastroenterol. 2016;51:774-81.

7. Collins AB, Pawlak R. Prevalence of vitamin B12 deficiency among patients with thyroid dysfunction. Asia Pac J Clin Nutr. 2016;25:221-6.

8. Calvete O, Reyes J, Zuñiga S, Paumard-Hernández B, Fernández $\mathrm{V}$, Bujanda L, et al. Exome sequencing identifies ATP4A gene as 
responsible of an atypical familial type I gastric neuroendocrine tumour. Hum Mol Genet. 2015;24:2914-22.

9. Calvete O, Varro A, Pritchard DM, Barroso A, Oteo M, Morcillo MÁ, et al. A knockin mouse model for human ATP4a R703C mutation identified in familial gastric neuroendocrine tumors recapitulates the premalignant condition of the human disease and suggests new therapeutic strategies. Dis Models Mech. 2016;9(9):975-84.

10. Livak KJ, Schmittgen TD. Analysis of relative gene expression data using real-time quantitative PCR and the $2^{-\triangle \Delta C T}$ method. Methods. 2001;25:402-8.

11. Yachdav G, Kloppmann E, Kajan L, Hecht M, Goldberg T, Hamp $\mathrm{T}$, et al. PredictProtein-an open resource for online prediction of protein structural and functional features. Nucleic Acids Res. 2014;42:W337-43.
12. Ho PWM, Goradia a, Russell MR, Chalk a M, Milley KM, Baker EK, et al. Knockdown of PTHR1 in osteosarcoma cells decreases invasion and growth and increases tumor differentiation in vivo. Oncogene. 2014. doi:10.1038/onc.2014.217.

13. Solter D, Solter M. Thyrotropin and thyroid hormone economy in euthyroid Hashimoto's thyroiditis. Acta Clin Croat. 2015;54:52-6.

14. Witrand A, Scaramuzzino S, Nigon D, Lukas C, Allanore Y, Vittecoq $\mathrm{O}$, et al. Association of polymorphisms on OPG, rank and RANKL with ACPA presence and erosions: results of a meta-analysis on 1570 rheumatoid arthritis patients from 3 French cohorts. Arthritis Rheumatol. 2014;66:S500-1.

15. Helmick CG, Felson DT, Lawrence RC, Gabriel S, Hirsch R, Kwoh CK, et al. Estimates of the prevalence of arthritis and other rheumatic conditions in the United States. Part I. Arthritis Rheum. 2008;58:15-25. 